for his many helpful suggestions throughout this work and to Dr. W. Nakahara for his invaluable advice on the anatomical examination. She is also indebted to the Keimei Society for financial assistance.

\title{
Sterilising Action of Acids on Putrifactive Bacteria, Bac. typhosus and Vib. cholerae.
}

First Report.-Sterilising action of Mineral Acids.

Sogo Tetsumoto.

(Received September 19, 1932.)

\section{Introduction.}

If we compare sterilising power of the same concentration of reagents, we know that acids have stronger sterilising power than bases or salts generally (1) (11). Owing to this fact, there are many studies about sterilising action of acids.

But before Krönig und Paul's foundamental studies about the sterilising action of reagents, many studies seem the observation of acid molecules only. Krönig and Paul said that $\mathrm{P}_{\mathrm{H}}$ are the essential part about sterilising action of acids and that anions and undissociated molecules have very weak or no sterilising power. After this study Bail's, Paul and Birstein's, and many other studies published one after another about this problem.

If we summarise all these studies untill now, are as follows:

(1) Sterilising action of acids depends on Pri almost totally.

(2) Anions or undissociated molecules have no sterilising power or have very weak sterilising power.

But according to my many experiments, of course $P_{H}$ is the essential part about the sterilising action of acids, following questions arouse;

(1) Are anions and undissociated molecules really weak, or almost no sterilising power?

(2) How are the sterilising power of each reagent under the important condition of the experiment e.g. (to experience always about certain quantities of the microorganisms having constant resisting power)? This condition is very important and that this has been treated carelessly by many experimenters.

(3) How are the collation of sterilising action at the same $\mathrm{Pr}_{\mathrm{r}}$ and at the same normal solution? 
(4) Possibility to find out harmless and effective sterilising chemicals and their utilization for drink and diet.

To study and clear up more thoroughly on these questions I performed following experiments. I wish to express my profound thanks to Dr. S. Kojima for his kind leading about my study.

\section{Experiment.}

(1) Inoculation of microorganisms.

Taking a sufficient quantity of microorganisms to ascertain their life and death, and then to cause the loss of the toxic action of reagent upon bouillon, I inoculated one platinum loopful of culture: the diameter of the loop was $4 \mathrm{~mm}$., having the diameter of platinum wire $0.6 \mathrm{~mm}$.

(2) Mineral acids used.

$\mathrm{HNO}_{3}$, IHCl, $\mathrm{H}_{2} \mathrm{SO}_{4}, \mathrm{H}_{3} \mathrm{PO}_{4}$ (ortho), Cromic asid (anhydride), Osmic acid (Osmium letra oxide), $\mathrm{HCNO}, \mathrm{H}_{3} \mathrm{BO}_{3}$.

(3) Species of microorganisms used for the experiments.

\begin{tabular}{l|c|c}
\hline \multicolumn{1}{c|}{ Name } & gram & Mobility \\
\hline Staphylococcus pyogenes aureus & + & - \\
Bacillus typhosus & - & + \\
Proteus vulgaris Hauser & + & + \\
Vibrio cholerae & - & + \\
\hline
\end{tabular}

(4) Standard culture media.

For the purpose to keep the constant vital forces and resisting power for Staph. c. pyog. aur, Bac. typhosus, Prot. vulgaris, I used next bouillon and agar.

Standard Bouillon: Liebig's meat extract

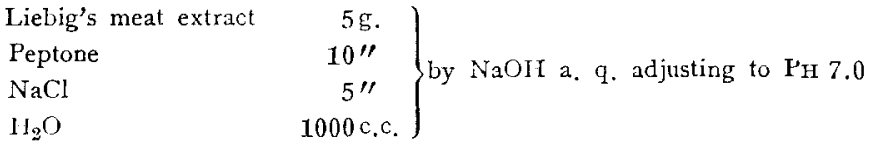

Standard agar: With above bouillon made 2.5\% agar slant.

For Vib. cholerae,

Standard Bouillon: Liebig's meat exlract

$\left.\begin{array}{lr}\text { Liebig's meat exlract } & 5 \mathrm{~g} . \\ \text { Peptone } & 10 " \\ \mathrm{NaCl} & 5 \% \\ \mathrm{H}_{2} \mathrm{O} & 1000 \mathrm{c} . \mathrm{c} .\end{array}\right\}$ by $10 \% \mathrm{Na}_{2} \mathrm{CO}_{3}$ adjusting to $\mathrm{PH}_{\mathrm{H}} 8.0$

Standard agar: With above bouillon made $2.5 \%$ agar slant.

(5) Standard resisting power of microorganisms used for experiments.

For 5 days, I repeated 24 hours bouillon cultures of Staph. c. pyog. aureus, Proteus vulgaris H., Bac. tyhosus and Vib. cholerae with standard bouillon, and fixed their respective vital forces. With pure phenol crystal 
(M. P. $40^{\circ} \mathrm{C}$ ) I made watery solution of $1 / 75,1 / 90,1 / 100,1 / 175$ dilution by weight.

According to G. Reddish's method ${ }^{(1: i)}$, I chose next group anong many cultivated microorganisms.

\begin{tabular}{|c|c|c|c|c|}
\hline \multicolumn{5}{|c|}{ Phenol solution } \\
\hline \multirow{2}{*}{$\begin{array}{l}\text { Surviving time } \\
\text { (minute) }\end{array}$} & $1 / 75$ dilution & 1/90 dilution & 1/100 dilution & $1 / 175$ dilution \\
\hline & Staph, coc. pyog. & Bac. typhos. & Prot, vulg. & Vib. chol. \\
\hline 5 & + & + & + & + \\
\hline 10 & + & \pm & \pm & \pm \\
\hline 15 & - & - & - & - \\
\hline
\end{tabular}

With these 4 species I made standard agar slant culture every day at $37^{\circ} \mathrm{C}$.

(6) Time.

From the put instant of the inoculation into bouillon, I set the reaction time as follows:

$1 \mathrm{~m}, 2.5,5,10,15,20,30,45,60,90,2 \mathrm{~h}, 3,6,9,12,24,36, \therefore \mathrm{t} . \dot{ }$

$$
\text { m.....minule h.....hour }
$$

New Process Tried On Chemical Sterilisation.

G. Reddish's method used for the chemical sterilisation, is as follows :

Reagent 10 c.c. +24 hours bouillon culture 1 c.c. of microorganism. This process is used generally now. But if we put 1 c.c. of bouillon culture into 10 c.c. of reagent, concentration and chemical characters of reagent will be greatly changed by it. Above all this fact is distinct when reagent is acid solution. For example;

\begin{tabular}{|c|c|c|c|c|c|c|}
\hline Reagent & Normal & $\mathrm{PH}$ & $\begin{array}{l}\text { PH ofter add- } \\
\text { ing } 1 \text { c.c. of } \\
\text { Bac. typhos. } \\
\text { bouillon cul- } \\
\text { ture }\end{array}$ & $\begin{array}{l}\text { PH after add- } \\
\text { ing } 1 \text { c.c. of } \\
\text { Vib. chol. } \\
\text { bouillon cul- } \\
\text { ture }\end{array}$ & $\begin{array}{l}\text { PH after add- } \\
\text { ing } 0.1 \text { c.c. of } \\
\text { Bac. typh. } \\
\text { bouillon cul- } \\
\text { ture }\end{array}$ & $\begin{array}{l}\text { PH after add- } \\
\text { ing } 0.1 \text { c.c. of } \\
\text { Vib. chol. } \\
\text { bouilion cul- } \\
\text { ture }\end{array}$ \\
\hline $\mathrm{HNO}_{3}$ & $N / 100$ & 2.0 & $2.8-3.0$ & $3.2-3.4$ & $2.1-2.2$ & $2.2-2.4$ \\
\hline $\mathrm{HCl}$ & $\prime \prime$ & "l & $\prime \prime$ & $\prime \prime$ & $\prime \prime$ & $\prime \prime$ \\
\hline $\mathrm{H}_{2} \mathrm{SO}_{4}$ & $\prime \prime$ & $" f$ & "I & $\prime \prime$ & " & " \\
\hline $\mathrm{H}_{3} \mathrm{PO}_{4}$ & " & 2.1 & $\prime \prime$ & $\prime \prime$ & $"$ & " \\
\hline $\mathrm{H}_{2} \mathrm{CrO}_{4}$ & $" \prime$ & 4.4 & $5.4-5.6$ & $5.8-6.0$ & $4.6-4.8$ & $4.8-5 \cdot 0$ \\
\hline $\mathrm{H}_{2} \mathrm{OsO}_{4}$ & $1 / 200,000$ & 5.6 & $6.4-6.6$ & $6.6-6.8$ & 5.8 & $5.8-6.0$ \\
\hline $\mathrm{H}_{3} \mathrm{BO}_{3}$ & 1 Nor. & 4.8 & $5.6-5.8$ & $6.0-6.2$ & $5.0-5.2$ & $5.2-5.4$ \\
\hline $\mathrm{HNO}_{3}$ & $1 / 1,000$ & 3.0 & 4.0 & $4.2-4.4$ & 3.2 & $3.2-3.4$ \\
\hline $\mathrm{HCl}$ & $" \prime$ & " & " & "l & $\prime \prime$ & $\prime \prime$ \\
\hline $\mathrm{H}_{2} \mathrm{SO}_{4}$ & $\prime \prime$ & " & " & " & $"$ & $" \prime$ \\
\hline
\end{tabular}




\begin{tabular}{l|c|c|c|c|c|c}
$\mathrm{H}_{3} \mathrm{PO}_{4}$ & $\prime \prime$ & 3.2 & $4.4-4.6$ & 4.6 & 3.6 & 3.6 \\
$\mathrm{H}_{2} \mathrm{CrO}_{4}$ & $\prime \prime$ & 4.8 & $5.8-6.0$ & 6.0 & $5.0-5.2$ & $5.0-5.4$ \\
$\mathrm{H}_{2} \mathrm{OsO}_{4}$ & $\mathbf{1} / 1,000,000$ & 5.6 & $6.8-7.0$ & $6.8-7.2$ & 5.8 & $5.8-6.2$ \\
\hline
\end{tabular}

$\left(20^{\circ} \mathrm{C}\right)$

Namely if we put 1 c.c. of bouillon culture of Bac. typhosus into 10 c.c. of reagent, $\mathrm{P}$ i of reagent change 0.8-1.2. On Staph. c. pyog. and Proteus vulgar., we see also nearly the same results. On vib. choler., the change of $\mathrm{P}_{\mathrm{H}}$ is $1.2-1.6$. Even if we put 0.1 c.c. of bouillon culture of microorganism, into 10 c.c. of reagent, the change of $\mathrm{PH}$ is $0.1-0.4$ on Bac. typhosus, Prot. vulgaris and Staph. c. pyogenes, and 0.2-0.6 on Vib. cholerae. According to this respect Shimokawa ${ }^{(1)}$, Tsubouchi ${ }^{(5)}$ took such process that instead of bouillon culture 1 c.c. they attached microorganism to the silk thread or artificial wild-cocoon silk thread with certain mass and diameter, and put it into reagent. By this method change of reagent will be relatively small. But into such solution as pure dilute acid, silk thread or artificial wild cocoon silk thread will give some change on chemical character of reagent during relatively long time. Krönig and Paul ${ }^{(5)}$ adhered suspension of microorganisms to garnets having the certain size, and put these into reagents. By above methods microorganisms will be much near the sills thread or artificial wild-cocoon thread or garnet and will be not homogeneously distribut in reagents.

To avoid these defects, I tried new process; that is as follows:

I put 0.1 c.c. of bacterial suspension to 10 c.c. of reagent with the pippet measuring $1 / 100$ c.c. degree, to forbid nothing to enter into the reagent except bacterial suspension, and to avoid the change of chemical characters or concentration of reagent.

Bacterial suspension :-

Put $2 \mathrm{mg}$. of colony of 24 hours standard agar slant culture of microorganism into 10 c.c. of the sterilised physiological $\mathrm{NaCl}$ solution and make homogeneous suspension.

By this method nothing can enter into reagents except the microorganism, and that total germs of 0.1 c.c. of bacterial suspension are as follows:

$\begin{array}{lr}\text { Bac. typhosus } & 20,000,000 \text { germs } \\ \text { Vib. choleraef } & 23,000,000 " ~ \\ \text { Proteus vulgaris Ilauser } & 28,000,000 " ~\end{array}$

The change of $\mathrm{Pr}$ are as follows: 


\begin{tabular}{|c|c|c|c|c|c|c|}
\hline Reagent & $\begin{array}{c}\text { Concentration } \\
\text { (Normal) }\end{array}$ & $P_{\mathrm{HI}}$ & $\begin{array}{l}\text { PH after add- } \\
\text { ing } 1 \text { c.c. of } \\
\text { Bac. typh. } \\
\text { suspension }\end{array}$ & $\begin{array}{l}\text { Pu after add- } \\
\text { ing } 1 \mathrm{c.c} \text {. of } \\
\text { Vib. chol. } \\
\text { suspension }\end{array}$ & $\begin{array}{l}\text { PH after add- } \\
\text { ing } 0.1 \text { c.c. of } \\
\text { Bac, typh. } \\
\text { suspension }\end{array}$ & $\begin{array}{l}\text { Pr after add- } \\
\text { ing } 0.1 \text { c.c. of } \\
\text { Vib. choler. } \\
\text { suspention }\end{array}$ \\
\hline $\mathrm{HNO}_{3}$ & $1 / 100$ & 2.0 & 2.2 & $2.2-2.4$ & 2.0 & 2.0 \\
\hline $\mathrm{HCl}$ & " & $" \prime$ & $"$ & $" \prime$ & $" \prime$ & " \\
\hline $\mathrm{H}_{2} \mathrm{SO}_{4}$ & " & $" \prime$ & " & " & $"$ & " \\
\hline $\mathrm{H}_{3} \mathrm{PO}_{4}$ & $" \prime$ & 2.1 & 2.4 & 2.4 & 2.1 & 2.1 \\
\hline $\mathrm{H}_{2} \mathrm{CrO}_{4}$ & $"$ & 4.4 & 4.6 & $4.6-5.0$ & 4.4 & 4.4 \\
\hline $\mathrm{H}_{2} \mathrm{OsO}_{4}$ & $1 / 200,000$ & 5.6 & 5.8 & $5.8-6.0$ & 5.6 & 5.6 \\
\hline $\mathrm{H}_{3} \mathrm{BO}_{3}$ & 1 & 4.8 & 5.0 & $5.0-5.2$ & 4.8 & 4.8 \\
\hline $\mathrm{HNO}_{3}$ & $1 / 1,000$ & 3.0 & 3.2 & $3.2-3.4$ & 3.0 & 3.0 \\
\hline $\mathrm{HCl}$ & " & $" \prime$ & $"$ & " & " & " \\
\hline $\mathrm{H}_{2} \mathrm{SO}_{4}$ & $"$ & $" \prime$ & $"$ & $"$ & $"$ & $" \prime$ \\
\hline $\mathrm{H}_{3} \mathrm{PO}_{4}$ & " & 3.2 & $3.4-3.6$ & $3.4-3.6$ & 3.2 & 3.2 \\
\hline $\mathrm{H}_{2} \mathrm{CrO}_{4}$ & $" \prime$ & 4.8 & $5.0-5.2$ & $5.0-5.4$ & 4.8 & 4.8 \\
\hline $\mathrm{H}_{2} \mathrm{OsO}_{4}$ & $1 / 1,000,000$ & 5.6 & $5.8-6.0$ & $5.8-6.2$ & 5.6 & 5.6 \\
\hline
\end{tabular}

Namely if I put 0.1 c.c. of suspension of Bac. typhosus, or Vib. cholerae, into 10 c.c. of reagent, there is almost no change about $P_{r 1}$ of the reagent. On Staph. c. pyogenes and Proteus vulgaris Hauser, I saw the same result.

Performance of the experiment :-

Take $2 \mathrm{mg}$. from each colonies of 24 hours standard agar slant culture of Staph. c. pyog. aureus, Proteus vulgaris Hauser, Bac. typhosus and Vib. cholerae, and put each $2 \mathrm{ng}$. into 10 c.c. of sterilising physiological $\mathrm{NaCl}$ solution and make 4 microorganic suspensions. Make these 4 species of suspensions at $20^{\circ} \mathrm{C}$ in incubator (difference within $0.5^{\circ} \mathrm{C}$ ). Also keep each series of 10 c.c. taken from reagents at $20^{\circ} \mathrm{C}$. Put 0.1 c.c. of the suspension into 10 c.c. of reagent and mixed homogeneously. At the every certain time I inoculate microorganisms from reagents into standard bouillon with the certain platinum loop and cultivate them $48-72$ hours in incubator at $37^{\circ} \mathrm{C}$. Alive or death of microorganisms are determined by the turbidity of standard bouillon culture. When the result is doubtful 1 repeat the experiment many times, and then I ascertained by morphological investigations and the aggiutination test with rabbit's immune serum of each microorganisms.

Results are following tables:

Experimental results : -

Table No. 1. Weight $\%$ and $\mathrm{PH}_{\mathrm{H}}$ at $N / 100, N / 1000$, of $\mathrm{HNO}_{3}, \mathrm{HCl}, \mathrm{H}_{2} \mathrm{SO}_{4}$, $\mathrm{H}_{3} \mathrm{PO}_{4}$ (ortho), Cromic acid, HCNO, 1 Nor. of $\mathrm{H}_{3} \mathrm{BO}_{3}, N / 100,000$, $N / 1,000,000$ of Osmic acid.

Table No. 2. 3. Sterilising action at $N / 100, N / 1000$, except boric acid, Boric acid...... 1 Nor. 
Table No. 4. Sterilising action of dilute cromic acid and extremly dilute solution e. g. $N / 100,000, N / 1,000,000$ of osmic acid.

Table No. 5. Relation between $\mathrm{P}_{\mathbf{H}}$ and normal concentration of mineral acids which affect on sterilisation.

Table No. 6. Sterilising action of anion of each mineral acids.

To ascertain the sterilising action of anion of mineral acids, 1 made alkaline salts having the same anion of each acids. For Staph. coc. pyogen, Prot. vulgar. and Bac. typhos. $N / 100$ of alkaline salts of each acids and $N / 100,000$ of alkaline osmium salt, 1 nor. of alkaline boric acid salt, are used. For Vib. choler. $N / 1,000$ of each alkaline salts, $N / 1,000,000$ of alkaline salt of osmic acid, $N / \overline{5}, 000$ of alkaline salt of cromic acid, 1 nor. of alkaline salt of boric acid are used. Each of $\mathrm{K}$ and $\mathrm{Na}$ salts give nearly the same results, so 1 denote the results of $\mathrm{K}$-salt only.

Table No. 1. Weight $\%$ and $\mathrm{PH}_{\mathrm{H}}$ of reagents.

\begin{tabular}{l|c|c|c|c|c|c|c}
\hline \hline Reagents & $\begin{array}{c}\text { Molecul. } \\
\text { weight }\end{array}$ & $\begin{array}{c}\text { Concent. } \\
\text { (Normal) }\end{array}$ & $\mathrm{PH}_{\mathrm{H}}$ & Weight \% & $\begin{array}{c}\text { Concent. } \\
\text { (Normal) }\end{array}$ & PH & Weight \% \\
\hline $\mathrm{HNO}_{3}$ & 63.018 & $1 / 100$ & 2.0 & 0.063 & $1 / 1,000$ & 3.0 & 0.0063 \\
$\mathrm{HCl}$ & 36.458 & $\prime \prime$ & $\prime \prime$ & 0.037 & $\prime \prime$ & $\prime \prime$ & 0.0037 \\
$\mathrm{H}_{2} \mathrm{SO}_{4}$ & 98.016 & $\prime \prime$ & $\prime \prime$ & 0.049 & $\prime \prime$ & $\prime \prime$ & 0.0049 \\
$\mathrm{H}_{3} \mathrm{PO}_{4}$ & 98.064 & $\prime \prime$ & 2.14 & 0.033 & $\prime \prime$ & 3.2 & 0.0033 \\
$\mathrm{CrO}_{3}$ & 100.01 & $\prime \prime$ & 4.4 & 0.050 & $\prime \prime$ & 4.8 & 0.0050 \\
$\mathrm{OsO}_{4}$ & 255.0 & $1 / 100,000$ & 5.4 & 0.000128 & $1 / 1,000,000$ & 5.6 & 0.000013 \\
$\mathrm{HCNO}$ & 43.021 & $1 / 100$ & 4.4 & 0.043 & $1 / 1,000$ & 4.6 & 0.0043 \\
$\mathrm{H}_{3} \mathrm{BO}_{3}$ & 61.924 & 1 Nor. & 4.8 & 2.064 & & & \\
\hline
\end{tabular}

Table No. 2. Sterilising action at $N / 100$ solution.

\begin{tabular}{|c|c|c|c|c|c|c|c|c|c|c|c|c|c|c|c|c|c|c|c|c|c|}
\hline \multirow{3}{*}{$\begin{array}{c}\text { Name of microorg. } \\
\text { Surviving period }\end{array}$} & \multicolumn{5}{|c|}{$\begin{array}{l}\text { Staph. c. } \\
\text { pyog. }\end{array}$} & \multicolumn{6}{|c|}{ Prot, vulgar. } & \multicolumn{5}{|c|}{ Bac. typhos. } & \multicolumn{5}{|c|}{ Vib. choler. } \\
\hline & & & & & $12 x$ & & & & & & $n$ & & & & & $\mathrm{~m}$ & & & & & $\mathrm{~m}$ \\
\hline & 1520 & 30 & 456 & 60 & \begin{tabular}{l|l}
0 & 1
\end{tabular} & 1 & 2.5 & 51 & 101 & & 02 & 2.5 & 51 & 015 & 203 & 30 & 12 & 2.5 & 5203 & & 45 \\
\hline $\mathrm{HNO}_{3}$ & ++ & + & - & - & -- & + & + & $+=$ & \pm & - & - & + & ++ & + \pm & - & - & - & - & -- & - & - \\
\hline $\mathrm{HCl}$ & ++ & + & - & - & -- & + & + & $+1=$ & \pm & -- & - & + & $+t$ & + \pm & - & - & - & - & $-\infty$ & - & $\rightarrow$ \\
\hline $\mathrm{H}_{2} \mathrm{SO}_{4}$ & ++ & + & - & - & - & + & + & $+=$ & \pm & - & - & + & +- & + \pm & - & - & - & - & -- & - & - \\
\hline $\mathrm{H}_{3} \mathrm{PO}_{4}$ & ++ & + & \pm & - & - & + & + & $+1+$ & + & - & - & + & +1 & ++ & + & - & \pm & - & -- & - & - \\
\hline $\mathrm{H}_{2} \mathrm{CrO}_{4}$ & ++ & - & - & - & -1 & + & \pm & -- & - & - & - & + & \pm- & --- & - & - & - & - &.-- & - & - \\
\hline $\mathrm{H}_{2} \mathrm{OsO}_{4}$ & -- & - & - & - & -- & - & - & - & - & - & - & - & -- & -- & - & - & - & - & -- & - & - \\
\hline HCNO & ++ & - & - & - & -- & + & - & - & - & - & - & + & +- & -- & - & - & - & - & -- & - & - \\
\hline $\mathrm{H}_{3} \mathrm{BO}_{3}$ & ++ & + & + & + & + & + & + & + & + & + & + & + & + & ++ & + &.+ & + & + & ++ & \pm & - \\
\hline Control & ++ & + & + & + & + & + & + & + & + & + & + & + & ++ & ++ & + & +1 & + & + & +1 & + & + \\
\hline
\end{tabular}


Table No. 3. Sterilising action at $N / 1000$ solution.

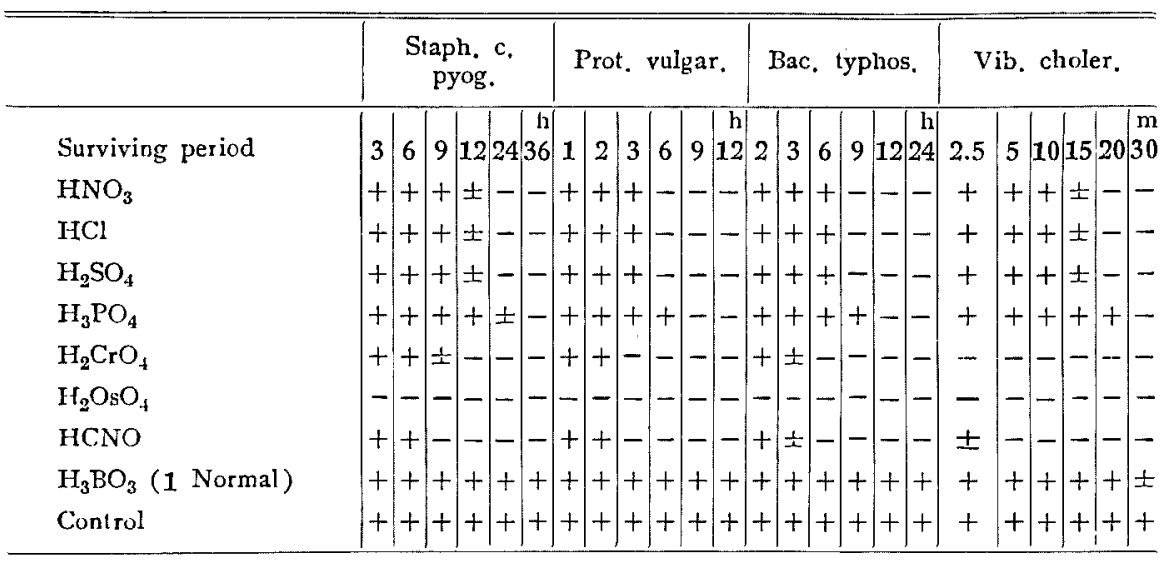

Table No. 4. Sterilising action of dilute Cromic acid and extremely dilute solution of Osmic acid.

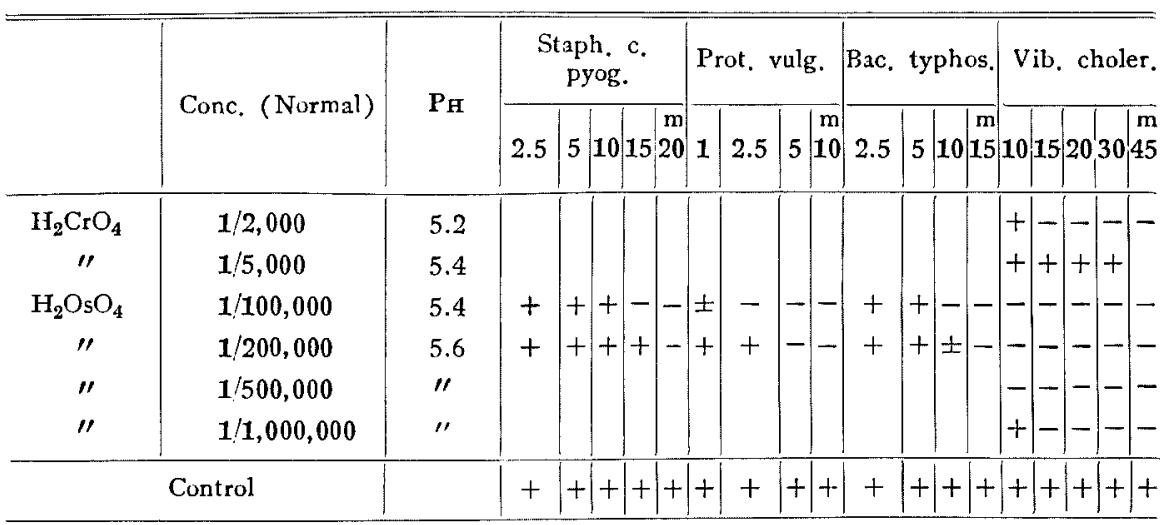

Table No. 5. Sterilising action of the same $P_{\boldsymbol{H}}$ of mineral acids, such as $\mathrm{HNO}_{3}, \mathrm{HCl}, \mathrm{H}_{2} \mathrm{SO}_{4}, \mathrm{H}_{3} \mathrm{PO}_{4}$.

\begin{tabular}{|c|c|c|c|c|c|c|c|c|c|c|c|c|c|c|c|c|}
\hline \multirow[b]{2}{*}{$\mathrm{PH} 2.0$} & \multicolumn{4}{|c|}{ Staph, c. pyogen. } & \multicolumn{4}{|c|}{ prot. vulgar. } & \multicolumn{4}{|c|}{ Bac. typhos. } & \multicolumn{4}{|c|}{ Vib. Choler. } \\
\hline & 15 & 20 & 30 & $\begin{array}{c}\mathrm{m} \\
45\end{array}$ & 2.5 & 5 & 10 & $\begin{array}{r}m \\
15\end{array}$ & 5 & 10 & 15 & $\begin{array}{c}\mathrm{m} \\
20\end{array}$ & 1 & 2.5 & 5 & $\begin{array}{c}m \\
10\end{array}$ \\
\hline $\mathrm{HNO}_{3}$ & + & + & + & - & + & + & \pm & - & + & + & \pm & - & - & - & - & - \\
\hline $\mathrm{HICl}$ & + & + & + & - & + & + & \pm & - & + & + & \pm & - & - & - & - & - \\
\hline $\mathrm{H}_{2} \mathrm{SO}_{4}$ & + & + & + & - & + & + & \pm & - & + & + & \pm & - & - & - & - & - \\
\hline $\mathrm{IH}_{3} \mathrm{PO}_{4}$ & + & + & + & - & + & + & \pm & - & + & + & \pm & - & - & - & - & - \\
\hline
\end{tabular}




\begin{tabular}{|c|c|c|c|c|c|c|c|c|c|c|c|c|c|c|c|c|}
\hline \multirow[b]{2}{*}{$\mathrm{PH}_{\mathrm{H}} 3.0$} & \multicolumn{4}{|c|}{ Staph. .. pyogen. } & \multicolumn{4}{|c|}{ Prot, vulgar. } & \multicolumn{4}{|c|}{ Bac. typhos. } & \multicolumn{4}{|c|}{ Vib. choler. } \\
\hline & 6 & 9 & 12 & $24^{\mathrm{h}}$ & 2 & 3 & 6 & $9^{\mathrm{h}}$ & 3 & 6 & 9 & $\frac{{ }^{h}}{12}$ & 10 & 15 & 20 & $\begin{array}{c}\mathrm{m} \\
30\end{array}$ \\
\hline $\mathrm{HNO}_{3}$ & + & + & \pm & - & + & + & - & - & + & + & - & - & + & \pm & - & $\overline{-}$ \\
\hline $\mathrm{HCl}$ & + & + & \pm & - & + & + & - & - & + & + & - & - & + & \pm & - & - \\
\hline $\mathrm{H}_{2} \mathrm{SO}_{4}$ & + & + & \pm & - & + & + & - & - & + & + & - & - & + & \pm & - & - \\
\hline $\mathrm{H}_{3} \mathrm{PO}_{4}$ & + & + & \pm & - & + & + & - & - & + & + & - & - & + & \pm & - & - \\
\hline
\end{tabular}

Table No. 6. Sterilising action of anions of mineral acids.

\begin{tabular}{|c|c|c|c|c|c|c|c|c|c|c|c|c|c|c|c|c|c|c|c|c|c|c|c|}
\hline & \multicolumn{5}{|c|}{$\begin{array}{l}\text { Staph. c. } \\
\text { pyog. }\end{array}$} & \multicolumn{5}{|c|}{ Prot. vulgar. } & \multicolumn{6}{|c|}{ Bac. typhos. } & \multicolumn{7}{|c|}{ Vib. choler. } \\
\hline Surviving period & $\left|\begin{array}{c}\mathrm{m} \\
10\end{array}\right|$ & $\begin{array}{c}\mathrm{m} \\
20\end{array}$ & $\begin{array}{l}d \\
6\end{array}$ & 7 & $\mid \begin{array}{c}d \\
8\end{array}$ & 2.5 & 5 & \begin{tabular}{c|c}
$\mathrm{m}$ & $\mathrm{d}$ \\
10 & 4
\end{tabular} & 5 & $\begin{array}{l}d \\
6\end{array}$ & & 15 & \begin{tabular}{|l|l}
$\mathrm{m}$ & \\
20 & 5
\end{tabular} & & 6 & $\begin{array}{l}\mathrm{d} / \\
7\end{array}$ & 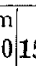 & & 30 & & 6 & & $\begin{array}{r}\mathrm{h} \\
12\end{array}$ \\
\hline $\mathrm{KNO}_{3}$ & ++ & + & $+t$ & + & + & + & + & +1 & -1 & + & + & + & $+1+$ & + & + & + & $t+$ & + & + & + & +- & & + \\
\hline $\mathrm{KCl}$ & ++ & + & + & + & + & + & + & ++ & + & + & + & + & $+1+$ & + & + & + & $+t$ & + & + & + & +- & $+t$ & + \\
\hline $\mathrm{K}_{2} \mathrm{SO}_{4}$ & ++ & + & + & + & + & + & + & ++ & -+ & + & $-1+$ & + & $+t$ & + & +- & + & ++ & $-1+$ & + & + & +- & $+t$ & + \\
\hline $\mathrm{K}_{3} \mathrm{PO}_{4}$ & ++ & + & + & + & + & + & + & ++ & + & + & + & + & +1 & + & + & + & $t+$ & -1 & + & + & + & $+t$ & + \\
\hline $\mathrm{K}_{2} \mathrm{CrO}_{4}$ & ++ & + & + & \pm & - & + & + & + \pm & $=-$ & - & $-t$ & + & $+1+$ & + & \pm & - & $+t$ & + & + & $+=$ & \pm . & -- & - \\
\hline $\mathrm{K}_{2} \mathrm{O}_{5} \mathrm{O}_{4}$ & ++ & - & - & - & - & + & + & -- & - & 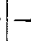 & $-1+$ & - & -- & - & - & 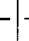 & $+t$ & - & - & - & - & -- & - \\
\hline $\mathrm{KCNO}$ & ++ & + & + & + & + & + & + & ++ & -1 & + & + & + & $+1+$ & + & + & t & $+t$ & + & + & + & + & $+t$ & + \\
\hline $\mathrm{K}_{3} \mathrm{BO}_{3}$ & ++ & + & + & + & \pm & + & + & ++ & \pm & - & + & + & $+1+$ & + & + & - & $+t$ & + & + & + & + & +- & - \\
\hline Control & ++ & + & \pm & - & - & + & + & + \pm & & & + & + & +1 & 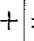 & \pm & & $+t$ & -+ & + & & + & & \pm \\
\hline
\end{tabular}

m.....minute

h.....hour

d......day.

\section{Summary.}

(1) Sterilising action of mineral acids such as $\mathrm{HNO}_{3}, \mathrm{HCl}, \mathrm{H}_{3} \mathrm{PO}_{4}$ is determined by $\mathrm{Pr}_{\mathrm{r}}$ of each solution, and has no relation about kind of acids.

(2) Anions of strong mineral acids have no sterilising action.

(3) There is no special relation between the value of molecular weight of mineral acids and sterilising action.

(4) On sterilising action of cromic acid and $\mathrm{HCNO}, \mathrm{PH}$ concerns a little.

(5) Sterilising action of dilute cromic acid and $\mathrm{HCNO}$, such as $N / 100$ are almost due to the action of undissociated acid molecule.

(6) The violent sterilising action of Osmic acid is chiefly due to the action of its anion and $\mathrm{Pr}$ concerns a little.

(7) Sterilising action of Boric acid is chiefly due to $\mathrm{PH}_{H}$ and the strilising action of it is very feeble.

\section{Reference}

(1) H. Shimokawa: Kokumin Eisei, Vol. 4, No. 3, 1925. 
(2) H. Yaoi : Zikken Igaku-zasshi, Vol. 8. No. 7, 1923.

(3) Y. Tohyama: Zikken Igaku-zasshi, Vol. 10. No. 9, 1925.

(4) S. Kozima., W. Nakagome: Nihonkoshiueisei-kyokai-zasshi, Vol. 6, No. 9 \& 11, 1930.

(5) N. Tubouchi: Kolkumin-Eisei, Vol, 8, No, 12, 1931.

(6) T. Hayashida: Kokumin-Eisei, Vol. 5, No. 12, 1928.

(7) M. Takeuchi: Kinsei Saikingaku Menekigaku, Vol, 2, 5 Edition, 1929.

( 8 ) B. Krönig, und Th. Paul: Zeitsch. f. Hyg. u. Infect., 25. 1-112, 1897.

(9) C. E. Winslow and E. E. Lochridge: Jour. of Infect., Dis. 3, 547-569, 1906.

(10) Th. Paul und G. Birstein: Bioch. Zeitsch., 29, 202-278, 1910.

(11) $\mathrm{Ph}$. Eisenberg: Centralbl. f. Bact., 82, 69-208, 1917.

(12) H. Friedenthal: Bioch. Zeitsch., 94, 47-68, 1919.

(13) G. F. Reddish: Amer. Jour. of Pub. Health, 18, 320-329, 1927.

(14) R. E. Buchnan and E. I. Fulmer: Physiol. \& Biochem, of Bact., Vol. II, 223-418, 1931. 